International Journal of Modern Physics D

(C) World Scientific Publishing Company

\title{
GRB ASTROPHYSICS IN THE SWIFT ERA AND BEYOND*
}

\author{
MICHAEL STAMATIKOS \\ Center for Cosmology and Astro-Particle Physics (CCAPP) Fellow/Department of Physics, \\ The Ohio State University, 191 West Woodruff Avenue, Columbus, OH 43210, USA \\ Michael.Stamatikos-1@nasa.gov
}

\begin{abstract}
Gamma-ray Bursts (GRBs) are relativistic cosmological beacons of transient high energy radiation whose afterglows span the electromagnetic spectrum. Theoretical expectations of correlated neutrino emission position GRBs at an astrophysical nexus for a metamorphosis in our understanding of the Cosmos. This new dawn in the era of experimental (particle) astrophysics and cosmology is afforded by current facilities enabling the novel astronomy of high energy neutrinos, in concert with unprecedented electromagnetic coverage. In that regard, GRBs represent a compelling scientific theme that may facilitate fundamental breakthroughs in the context of Swift, Fermi and IceCube. Scientific synergy will be achieved by leveraging the combined sensitivity of contemporaneous ground-based and satellite observatories, thus optimizing their collective discovery potential. Hence, the advent of GRB multi-messenger astronomy may cement an explicit connection to fundamental physics, via nascent cosmic windows, throughout the next decade.
\end{abstract}

Keywords: gamma-rays: bursts; radiation mechanisms: non-thermal; neutrinos.

\section{Electromagnetic Emission: The Synergy of Swift and Fermi}

The Swift MIDEX explorer mission 1 , comprised of the wide-field $(\sim 1.4 \mathrm{sr}$, halfcoded) hard X-ray (15-150 keV) Burst Alert Telescope (BAT), and the narrowfield (0.2-10 keV) X-Ray (XRT) and (170-600 nm) Ultraviolet-Optical (UVOT) Telescopes, has revolutionized our understanding of GRBs. The intrinsic multiwavelength instrumentation, coupled with a rapid ( $\lesssim 100$ seconds) autonomous slew capability, has ushered in an unprecedented era of source localization precision $\left(\lesssim 1^{\prime}-4^{\prime}\right)$ that is disseminated in real-time $(\lesssim 10$ seconds $)$ via the GRB Coordinate Network $(\mathrm{GCN})^{2}$, thus spear-heading international ground-based and satellite multi-wavelength follow-up campaigns. Swift's unique dynamic response and spatial localization precision, in conjunction with the aforementioned correlative ground-based follow-up efforts, have resulted in redshift determinations for $\gtrsim 133$ GRBs, including the most distant cosmological explosion, GRB 080913 at $z=6.695 \pm 0.025^{3}$, which has begun to constrain progenitor models 4 . Selection effects, such as detector composition and long accumulation timescales, bias BAT

*Contributed to the Proceedings of the 2nd Heidelberg Workshop: High-Energy Gamma-rays and Neutrinos from Extra-Galactic Sources (Max Planck Institute for Nuclear Physics). 
towards long, soft GRBs with lower characteristic photon energy $\left(\mathrm{E}_{\text {peak }}\right)$. Consequently, BAT GRBs comprise a separate statistical class, as is demonstrated by their fluence and redshift distributions $(\bar{z} \approx 2.3)$, from classical Burst and Transient Source Experiment (BATSE) GRBs. High-z GRBs afford an unprecedented opportunity to probe the earliest epoch of stellar formation via the detection of Population I, II (normal) and III (massive) stars. Meanwhile, the association of GRB 050509B with an elliptical host galaxy ${ }^{5}$ supports the idea that short GRBs may arise from compact binary mergers such as the coalescence of neutron stars $(\sim 1.4$ $\left.\mathrm{M}_{\odot}\right)$ with other neutron stars (DNS) or black holes $(\mathrm{BH}-\mathrm{NS} / \mathrm{BH}-\mathrm{BH}) \underline{6}$. With an estimated orbital lifetime of $\sim 15$ years, it is anticipated that Swift will continue to operate until $\sim 2017$.

Fermi, which is comprised of the $(<20 \mathrm{MeV}$ to $>300 \mathrm{GeV})$ Large Area Telescope (LAT) and the (10 keV - $30 \mathrm{MeV}$ ) Gamma-ray Burst Monitor (GBM), launched on June 11, 2008 and has an anticipated mission lifetime of $\sim 10$ years, taking it into $\sim 2018$. Fermi has already made some remarkable detections ${ }^{7}$ including the first GeV emission from a short burst (GRB 081024B) as well as the burst with the most isotropic energy release (GRB 080916C). The GBM, consisting of $12 \mathrm{NaI}$ (10-1000

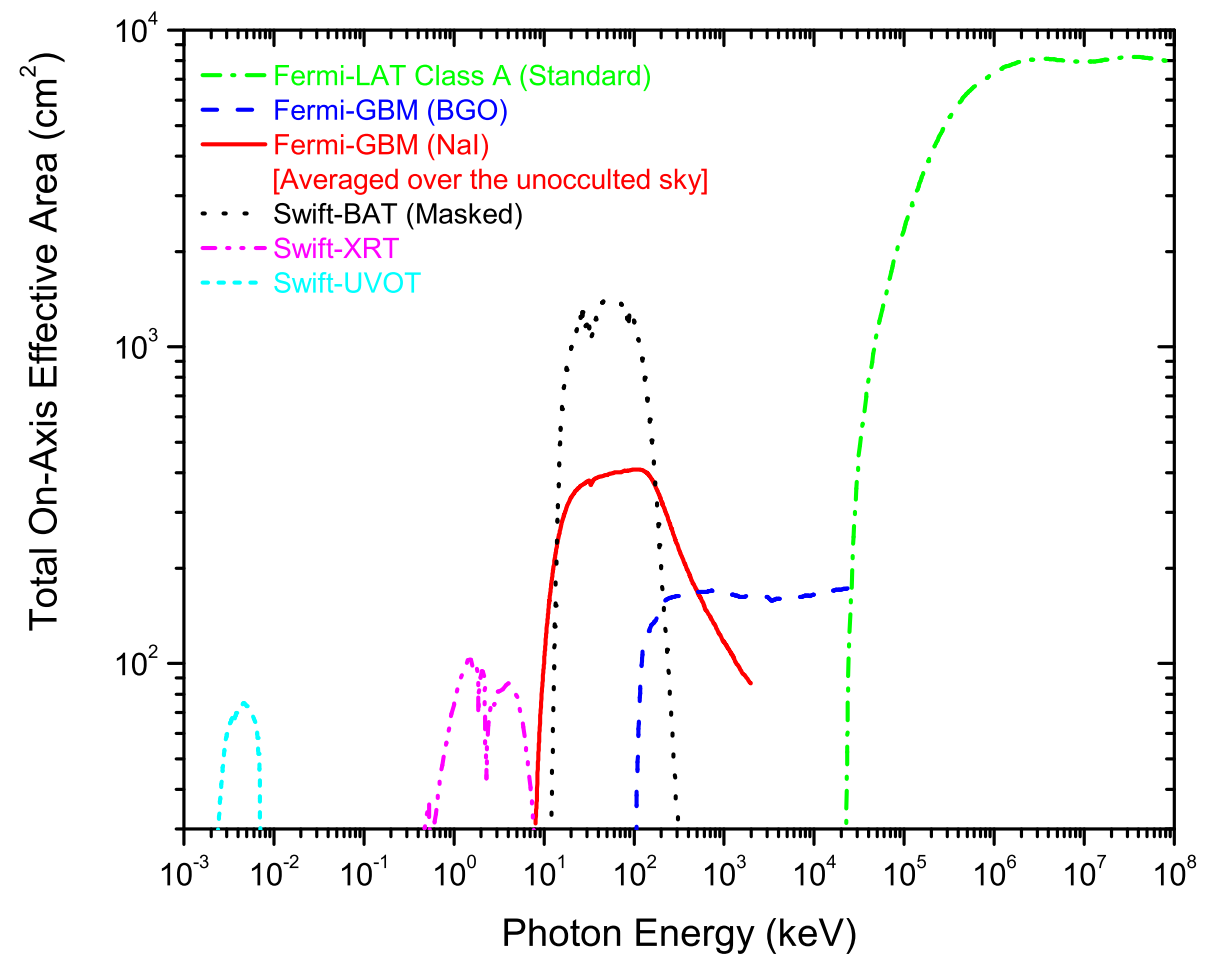

Fig. 1. Effective areas for Swift-BAT/XRT/UVOT and Fermi-GBM/LAT. Exceptional bursts may be detected over 11 energy decades facilitating temporal and spectral evolution studies. 
$\mathrm{keV})$ and 2 BGO (0.15-25 MeV) detectors, monitors $\sim 8$ steradians of the sky, and, in concert with LAT, enables Fermi to continuously span 7 energy decades, but not at the same sensitivity. As illustrated in Figure 1, Fermi's effective area drops by over $\sim 1.5$ orders of magnitude from LAT $(\mathrm{GeV})$ to GBM-NaI $(\mathrm{keV})$ energies, while the (masked) BAT $(\sim 20-100 \mathrm{keV})$ low energy effective area surpasses GBMNaI's by over a factor of $\sim 3$. Furthermore, although Swift has detected $\sim 400$ GRBs, the majority of $\overline{\mathrm{E}}_{\text {peak }} \sim 250 \mathrm{keV}$ values lie beyond BAT's canonical energy range. Thus, correlated observations would simultaneously augment Fermi-GBM's low energy response while increasing the number of $\mathrm{E}_{\text {peaks }}$ for Swift-BAT GRBs.

Additionally, since Swift's high fidelity localization precision surpasses GBM's by over $\sim 2-3$ orders of magnitude, BAT will facilitate GRB follow-up campaigns for Fermi bursts. In this manner, we expect that $\sim 35 \%$ of bursts within joint BAT-GBM analyses will be accompanied by panchromatic ground-based followup observations resulting in spectroscopic redshift determinations and host galaxy identifications. If a LAT GRB triggers Swift, chances favor subsequent detections by it's NFI's, since $\sim 95 \%$ of BAT GRBs are observed with XRT $(T \lesssim 200 \mathrm{ksec})$ and $\sim 60 \%$ have accompanied optical measurements from a combination of UVOT and ground-based observations. Hence, Fermi and Swift are poised to simultaneously span eleven decades in spectral energy (see Figure 1), and have already commenced correlated GRB observations for $\sim 25$ GRBs. It is anticipated that such joint studies will be possible a few times per month for an annual rate of $\sim 32 \pm 17 \mathrm{GRBs} 8$.

\section{Non-Electromagnetic Emission: A New Window with IceCube}

Thus far, a canonical phenomenological description, known as the fireball mode $\sqrt{9}$, has successfully described most prompt and afterglow GRB electromagnetic observations. However, despite great strides made by satellite-and ground-based electromagnetic observations, the details of GRB progenitor(s) remain concealed. This physical limitation is due to the initial optical thickness of the fireball, prior to adiabatic relativistic expansion, which precludes us from witnessing the genesis of a GRB. The relativisti@ nature of GRBs, inferred by the necessity for the expanding fireball shell to be optically thin, requires bulk Lorentz boost factors in the range $100 \lesssim \Gamma \lesssim 1000$, with typical values of $\sim 300$ for isotropic emission geometry. Since we are fundamentally limited by the optical opacity of the source at the early stages of its inception, perhaps the next evolution in our understanding of the microphysics of the central engine will be realized via the detection of non-electromagnetic emission.

The observed isotropic and cosmological spatial distribution of GRBs, coupled with the fact that the energy injection rates of ultra high-energy cosmic rays (UHECRs) and GRBs are similar, resulted in the phenomenological suggestion that GRBs may be the sources of UHECRs 11 . Canonical fireball phenomenology, in the context of hadronic (Fermi) acceleration within the astrophysical jet, predicts a taxonomy

${ }^{a}$ Apparent super-luminal motion has been observed in the radio afterglow of GRB 03032,10 . 
of correlated $\mathrm{MeV}$ to $\mathrm{EeV}$ neutrinos from GRBs of varying flavor and arrival times. Ideal for detection are $\sim \mathrm{TeV}-\mathrm{PeV}$ muon neutrinos 12 , which arise as the leptonic decay products of photomeson interactions $\left(p+\gamma \rightarrow \Delta^{+} \rightarrow \pi^{+}+[n] \rightarrow \mu^{+}+\nu_{\mu} \rightarrow\right.$ $e^{+}+\nu_{e}+\bar{\nu}_{\mu}+\nu_{\mu}$ ), within the internal shocks of the relativistic fireball. Since the prompt $\gamma$-rays act as the ambient photon target field, these neutrinos are expected to be in spatial and temporal coincidence, which imposes a constraint that is tantamount to nearly background-free searches $13 \mid 14$ in telescopes such as IceCube ${ }^{15}$.

\section{Multi-Messenger GRB Astronomy: Synthesis \& Science Impact}

GRBs are beacons for multi-messenger astronomy 16 that serve as astrophysical laboratories for Special/General Relativity, particle astrophysics and cosmology. In the electromagnetic regime, Swift's dynamic response and localization precision will complement prompt emission from Fermi, while facilitating ground-based follow-up via the GCN. Such unprecedented broad-band electromagnetic capability will enable joint GRB data sets that will enhance our understanding of burst parameter classifications, enable routine determinations of $\mathrm{E}_{\text {peak }}$ values, explore GRB emission geometry, and help test the viability of various redshift estimation methods 17 . In addition, a more accurate normalization between prompt and afterglow emissions will facilitate the determination of GRB energy budgets, while enabling investigations of spectral and temporal evolution 18 over unprecedented decades of energy.

The detection of non-electromagnetic emission from GRBs, via high-energy neutrinos with IceCube, has the potential for broad scientific breakthroughs. A positive detection of high-energy neutrinos would confirm hadronic acceleration in the relativistic GRB-wind providing critical insight to the associated micro-physics of the fireball while revealing an astrophysical acceleration mechanism for UHECRs, thus resolving a century old enigma. Given such tremendous discovery potential for science synergy and impact, our view of the Cosmos is bound to change forever.

\section{Acknowledgments}

M. Stamatikos is supported by a CCAPP Fellowship at the Ohio State University.

\section{References}

1. N. Gehrels et al., New Journal of Physics 9 (2007) 37.

2. S. Barthelmy, Astronomische Nachrichten 329 (2008) 340.

3. J. Greiner et al., The Astrophysical Journal 693 (2009) 1610-1620.

4. K. Belczynski et al., ArXiv e-prints: 0812.2470 (2008).

5. N. Gehrels et al., Nature 437 (2005) 851-854.

6. J. S. Bloom et al., The Astrophysical Journal 638 (2006) 354-368.

7. F. Longo et al., in Proceedings of the 2nd Heidelberg Workshop (2009).

8. M. Stamatikos et al., ArXiv e-prints: 0809.4724 (2008).

9. P. Mészáros and M. J. Rees, The Astrophysical Journal 405 (1993) 278-284.

10. G. B. Taylor et al., The Astrophysical Journal 609 (1993) L1-L4. 
11. E. Waxman, Physical Review Letters 75 (1995) 386-389.

12. E. Waxman and J. Bahcall, Physical Review Letters 78 (1997) 2292-2295.

13. M. Stamatikos et al., ArXiv e-prints: astro-ph/0510336 (2005).

14. M. Stamatikos et al., ArXiv e-prints: astro-ph/0602481 (2006).

15. A. Kappes et al., in Proceedings of the 2nd Heidelberg Workshop (2009).

16. M. Stamatikos et al., ArXiv e-prints: 0902.3022 (2009).

17. M. Stamatikos et al., in Proceedings of the 2007 GRB Conference (2008).

18. M. Stamatikos et al., ArXiv e-prints: 0902.0263 (2009). 